\title{
Minimally invasive direct lateral, retroperitoneal transforaminal approach for large L1-2 disc herniations with intraoperative CT navigational assistance: technical note and report of 3 cases
}

\author{
Adetokunbo A. Oyelese, MD, PhD, Jared Fridley, MD, David B. Choi, MD, Albert Telfeian, MD, PhD, \\ and Ziya L. Gokaslan, MD
}

Department of Neurosurgery, Rhode Island Hospital, Alpert Medical School of Brown University, Providence, Rhode Island

Upper lumbar (L1-2, L2-3) disc herniations are distinct in their diffuse presenting clinical symptomatology and have poorer outcomes with surgical intervention than those following mid and lower lumbar disc herniations and disc surgery. The authors present the cases of 3 patients with L1-2 disc herniations and significant stenosis of the spinal canal. The surgical approach used here combined the principles of transforaminal percutaneous endoscopic discectomy and the extreme lateral lumbar interbody fusion procedures with intraoperative CT-guided navigational assistance. The approach provides a safe corridor of direct visualization to the ventral thecal sac with minimal bony resection and could, in principle, reduce neurological injury and biomechanical instability, which likely contribute to poor outcomes at this level. https://thejns.org/doi/abs/10.3171/2017.11.SPINE17509

KEYWORDS minimally invasive spine surgery; image-guided spine surgery; AIRO intraoperative CT imaging system; transforaminal discectomy; XLIF approach; spinal navigation; surgical technique

$\mathrm{T}$ HE thoracolumbar and upper lumbar spine regions represent a transition segment juxtaposed between the flexible lordotic lumbar spine and the more rigid kyphotic thoracic spine. Thus, while the mobility of the lower lumbar segments makes this spinal region more susceptible to disc herniations, the frequency of such herniations in the upper lumbar spine (defined as L1-2 and L2-3) is much lower and has been reported to be on the order of $1 \%-2 \%$ of all lumbar disc herniations. ${ }^{3,9,10,23}$ Given the high density of neural elements located in this anatomical region (conus medullaris, upper cauda equina), the presenting symptoms in patients with upper lumbar disc herniations tend to be more varied than the clear-cut lumbosacral radiculopathy with which patients experiencing lower lumbar disc herniations present. ${ }^{6,723}$ Additionally, given the bony anatomical characteristics in this region with the more narrow, sagittally oriented facet joints, smaller spinal canal, and shorter width of the pars interarticularis, decompressive procedures geared toward the removal of central and large paracentral herniated disc fragments in the upper lumbar spine tend to be destabilizing, with additional fusion procedures required in twice as many patients than those who undergo the same treatment for lower lumbar level herniations. ${ }^{23}$ Not surprisingly, outcomes following surgical intervention for upper lumbar disc herniations tend to be less favorable than those for lower lumbar disc pathologies. ${ }^{23}$

Traditional approaches to central disc pathology in the upper lumbar spine include posterior laminectomy $y^{5,12,14}$ and posterolateral decompression with (transforaminal) interbody fusion. ${ }^{13,16,27}$ More recently, minimally invasive approaches including posterior percutaneous endoscopic transforaminal approaches for decompression ${ }^{2,8,26}$ and extreme lateral anterior approaches for interbody fusion have come into vogue. However, the former is geared more toward disc pathology laterally and paracentrally situated within the spinal canal, ${ }^{26}$ while the latter approach is designed more for removal of the anterior third of the disc for 


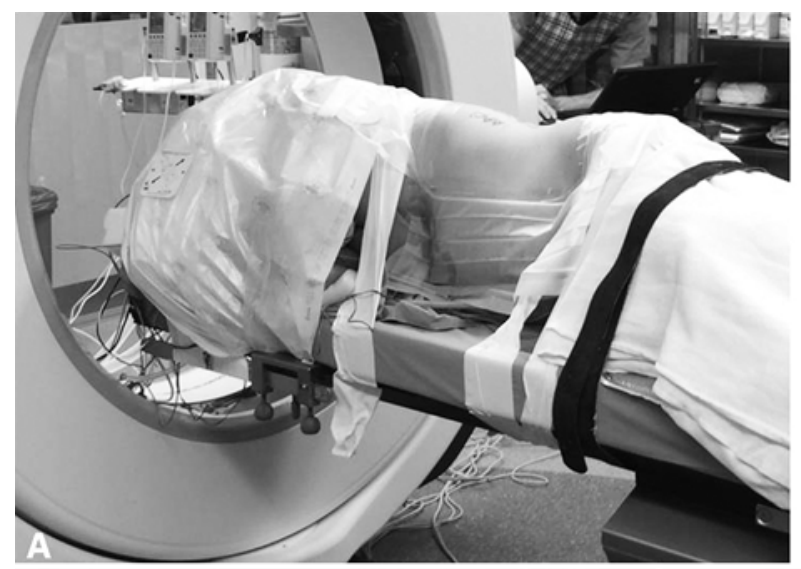

FIG. 1. A: Lateral positioning of the patient on the intraoperative radiolucent CT scanner table. B: Digital reference frame (lower arrow) mounted on the left posterior superior iliac spine and the Brainlab navigation probe (upper arrow) used to dissect through the psoas muscle and localize the L-1 foramen. C: Brainlab workstation screenshot showing navigationassisted localization of the L-1 foramen.
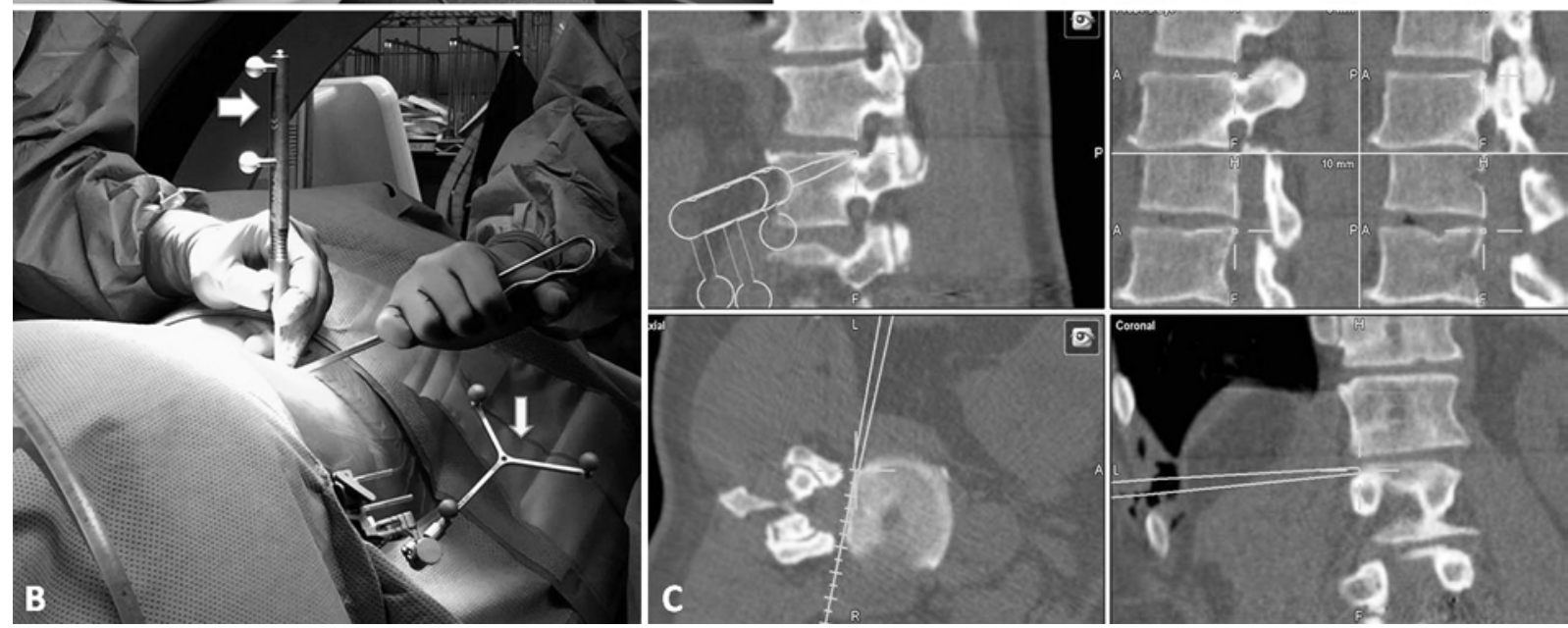

interbody fusion with indirect decompression of the neural elements by increasing disc and foraminal height. ${ }^{17,18} \mathrm{An}$ ideal approach to this region would adequately decompress the neural elements while maintaining the biomechanical integrity of the osseous elements of the upper lumbar spine.

Here, we report such an approach-a minimally invasive lateral, retroperitoneal, transpsoas, direct transforaminal approach to central and paracentral disc herniations at L1-2 in 3 patients presenting with intractable back pain, leg weakness, and near cauda equina syndrome, respectively. The approach combines the principles of the extreme or direct lateral, retroperitoneal transpsoas approach targeting the anterior third of the disc space and the posterior transforaminal approach through the neural foramen used in percutaneous endoscopic lumbar discectomy (PELD). We discuss the technical nuances involved in this approach and the significance of adjuncts used such as intraoperative CT imaging with navigational assistance.

\section{Surgical Technique}

After inducing general endotracheal anesthesia and establishing appropriate intravenous access, Foley catheter, and monitoring lines including those for electrophysiological monitoring, the patient was positioned in the right lateral decubitus position with an axillary roll under the right axilla on the radiolucent table (Trumpf Medical) for use with the intraoperative CT scanner (AIRO, Brainlab; Fig. 1A). Lateral fluoroscopy was used to localize the L1-2 disc space, and the skin was appropriately marked and then prepared and draped in standard surgical fashion. Two Schanz pins were inserted into the left posterior superior iliac spine to which a digital reference array clamp was attached. An intraoperative CT scan was obtained with autoregistration using the navigation system (Brainlab). A 3 - to 4-cm transverse skin incision was created in the left lateral flank directly over the posterior third of the L1-2 disc space, and the dissection was carried bluntly through the oblique and transverse abdominal muscles to expose the underlying 12th rib. The intercostal muscles were divided on the superior border of the rib, and the dissection was carried into the retroperitoneal space below the diaphragm. This approach, as well as the fact that the crural attachments of the diaphragm to the spine are situated more anteriorly on the vertebral body, allowed us to avoid mobilization of the diaphragm. The Brainlab navigation system was then used to direct the retroperitoneal dissection toward the neural foramen at L1-2 (Fig. 1B and C). The initial approach in the patients in cases 1 and 2 utilized an endoscope (joimax $\mathrm{GmbH}$ ) with an attached digital reference array for navigational assistance through a small tubular port (Fig. 2), but it was believed that greater visualization was needed for the large disc herniations. 

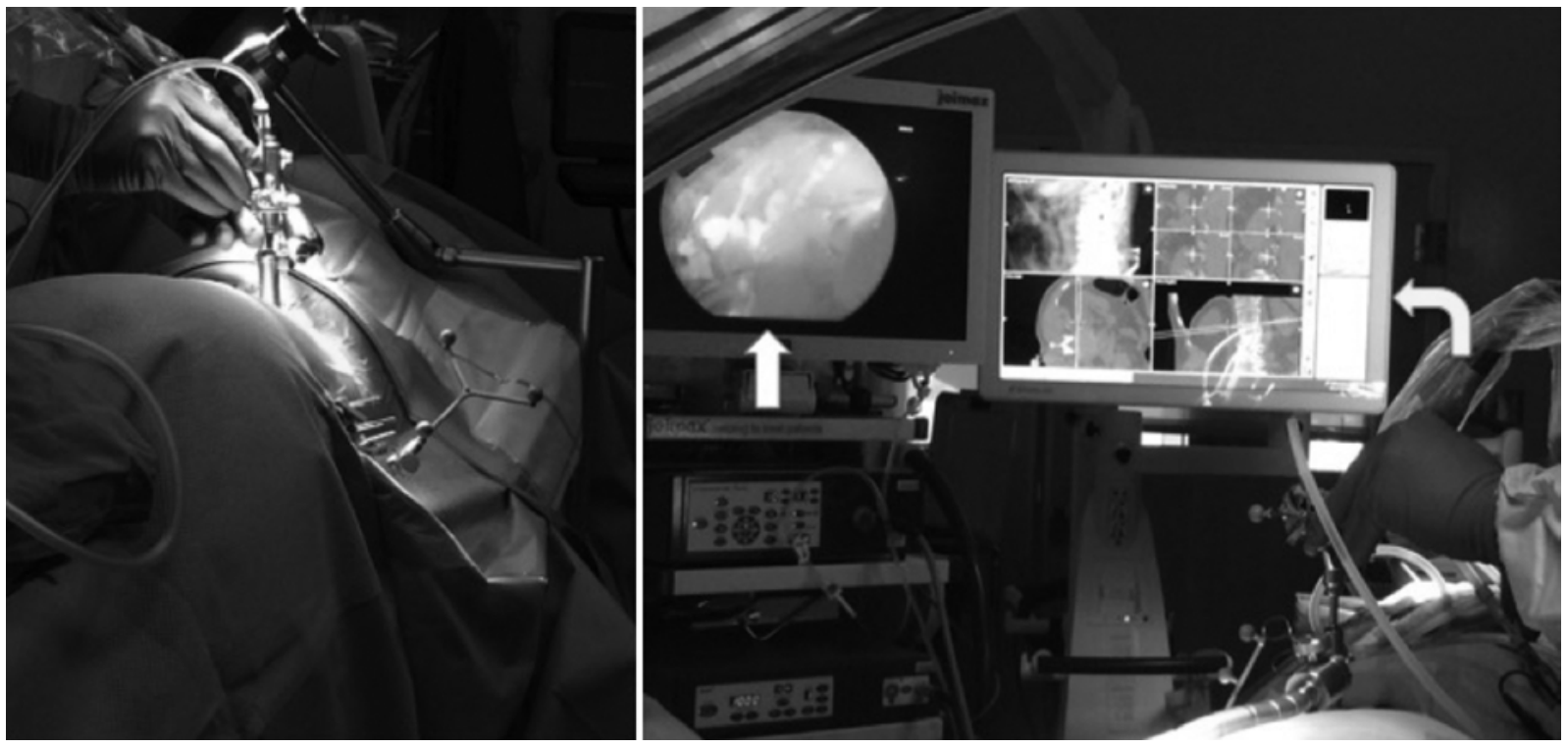

FIG. 2. A navigated joimax endoscope was used in the initial exposure. While the L1-2 disc was both visualized and localized with the endoscope (straight arrow) and navigation (curved arrow), a wider field of view was believed to be necessary given the size of the herniation and degree of canal stenosis; thus, an expandable lateral minimally invasive retractor was inserted for use with an operative microscope (not shown).

Serial minimally invasive dilators were inserted over a guidewire inserted into the posterior disc space over which a lateral minimally invasive expandable retractor (Globus or NuVasive) was placed with visualization of the psoas muscle at the border of the posterior vertebral body. The neural foramen was identified with navigational assistance, and the overlying psoas muscle was dissected free, exposing the underlying pedicle. Working between the exiting L-1 nerve root and the L-2 pedicle from a direct lateral approach, a foraminoplasty was performed by removing the superior portion of the L-2 pedicle and the ventrocaudal portion of the L-2 superior articular process with a navigated 4-mm diamond burr drill under microscopic guidance (Fig. 3A). In the patient in case 3, the disc was partially calcified; therefore, 5-mm troughs were drilled on either side of the disc space into the vertebral bodies of L-1 and L-2 just ventral to the disc annulus and extending to the contralateral pedicle. The disc annulus was incised, and disc fragments were removed directly from within the spinal canal by using straight and curved semi-bending flexible graspers. Ball-tipped nerve root dissectors were used to explore the spinal canal in a cephalad and caudad direction ventral to the thecal sac, with the removal of further disc fragments. In cases 1 and 3, large free fragments of disc were retrieved ventral to the thecal sac (Fig. 3B and Video 1).

VIDEO 1. Clip demonstrating key steps in the procedure. Copyright Adetokunbo A. Oyelese. Published with permission. Click here to view.

A large free fragment of disc was retrieved from the medial aspect of the L-2 pedicle in case 2 in which a more extensive foraminoplasty had been performed. The thecal sac, which had been displaced by the disc, could now be properly visualized, and in all 3 cases was free from compression (Fig. 3C). The navigation probe was then used to ensure the decompression had been carried all the way across the canal to the contralateral pedicle. The retractor was then withdrawn, and the wound was closed in layers.

\section{Case Reports}

\section{Clinical Presentation}

The patient in case 1 was a 66-year-old man with a history of lumbar spondylosis and chronic low-back pain who experienced an acute exacerbation with neurogenic claudication. Magnetic resonance imaging of the lumbar spine showed a large central L1-2 disc herniation and a residual spinal canal of $3 \mathrm{~mm}$ (Fig. 4A and B). He had no weakness or bladder and bowel dysfunction but rated his pain as a $6-10$ on a visual analog scale (VAS) of $0-10$ (Table 1). The
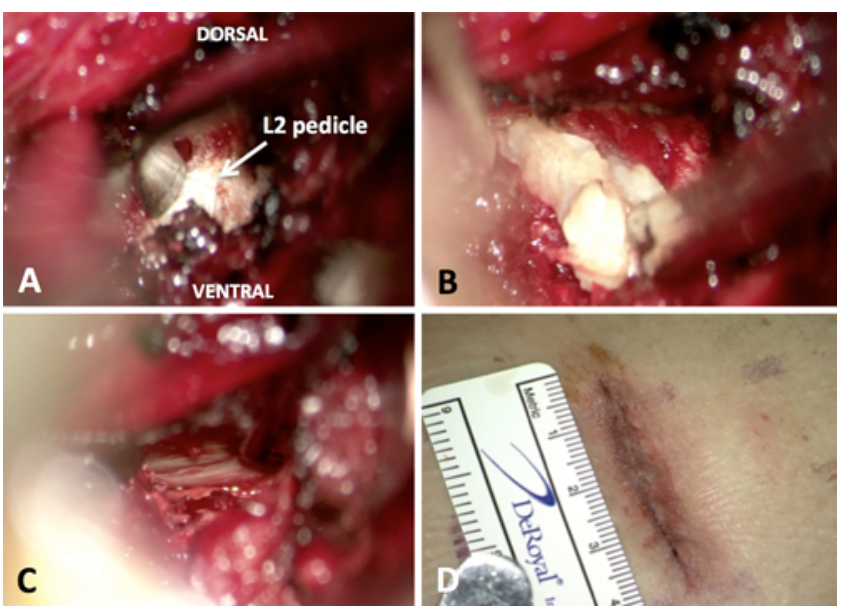

FIG. 3. A: Dissecting probe at the leading superior edge of the $L-2$ pedicle (arrow) showing pedicle resection during foraminoplasty. B: Removal of disc fragments from the canal. C: Ventral thecal sac visible after discectomy. D: Final closure of the $3-\mathrm{cm}$ minimally invasive incision. 


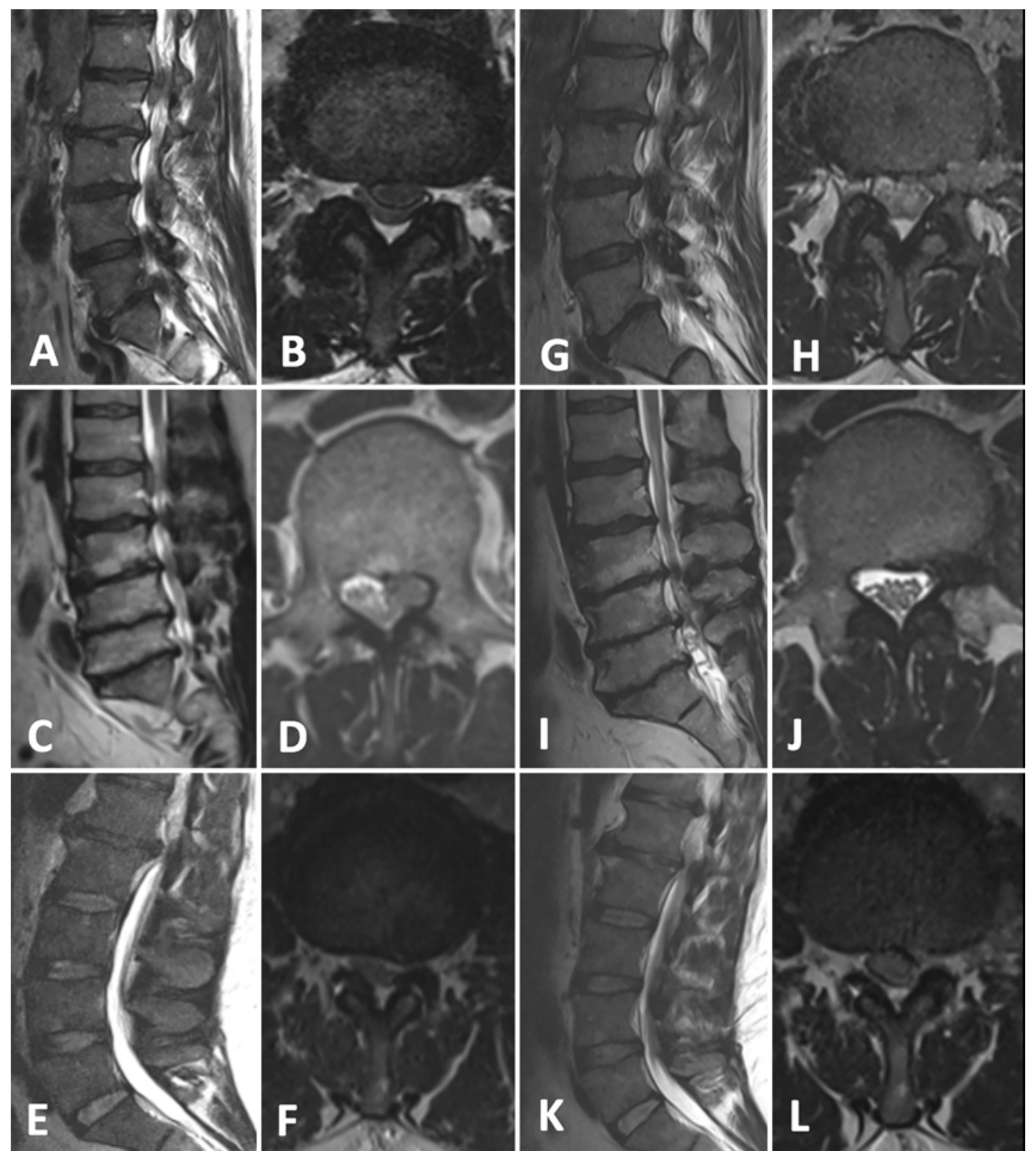

FIG. 4. Preoperative $(\mathbf{A}-\mathbf{F})$ and postoperative $(\mathbf{G}-\mathbf{L})$ axial and sagittal T2-weighted MR images showing resolution of disc herniation and improved patency of the spinal canal following surgery. In the preoperative images, note the large central disc herniation in cases 1 and 2 and the left free fragment herniation in case 2. The postoperative scans from the patient in case $3(K-L)$ were obtained 48 hours after surgery, too early for resolution of the postoperative changes, hence the less dramatic change from the preoperative scans $(E-F)$. Compare changes from preoperative to postoperative in case $1(A, B, G, H)$ and case $2(C, D, I, J)$.

pain radiated into the legs bilaterally with greater pain on the left. He had exhausted his conservative management options including physical therapy and epidural steroid injections. After careful consideration, surgical intervention for the L1-2 disc herniation was recommended.

The patient in case 2 was a 59-year-old man with a longstanding history of low-back pain who was now presenting with excruciating left anterior thigh pain that progressed to numbness and weakness with hip flexion over a 3-week period. An MRI study of the lumbar spine revealed a large L1-2 disc herniation with a free fragment lodged medial to the left L-2 pedicle (Fig. 4C and D).
The patient in case 3 was a 33-year-old man who suffered an injury while at work with continued intractable back pain unresponsive to conservative management. He also developed weakness and numbness in the legs, the left worse than the right, with intractable pain. He was admitted to the hospital for pain control and underwent MRI of the lumbar spine, which showed a central and left paracentral disc herniation with severe stenosis (Fig. 4E and F). The patient experienced an increase in pain, saddle anesthesia, and bilateral leg weakness including a left partial foot drop while in the hospital and therefore surgery was recommended. 
TABLE 1. Demographic, clinical, and operative parameters

\begin{tabular}{|c|c|c|c|c|}
\hline Parameter & Case 1 & Case 2 & Case 3 & Mean \\
\hline Age (yrs) & 66 & 59 & 33 & 52.67 \\
\hline Sex & Male & Male & Male & \\
\hline Presenting symptoms & $\begin{array}{l}\text { Low-back pain, neurogenic } \\
\text { claudication }\end{array}$ & $\begin{array}{l}\text { Low-back pain, It ant thigh pain \& } \\
\text { numbness, It hip flexor weakness }\end{array}$ & $\begin{array}{l}\text { Low-back pain, bilat lower extremity } \\
\text { weakness \& numbness }\end{array}$ & \\
\hline OR time (mins) & 262 & 263 & 148 & 224.33 \\
\hline Blood loss (ml) & 20 & 80 & 40 & 46.67 \\
\hline $\mathrm{FU}$ (mos) & 21 & 6 & 8 & \\
\hline Postop complications & $\begin{array}{l}\text { Lt ant thigh \& groin numbness; } \\
\text { transient pain }\end{array}$ & $\begin{array}{l}\text { Transient It ant thigh pain; stable } \\
\text { numbness }\end{array}$ & $\begin{array}{l}\text { Transient It ant thigh numbness \& } \\
\text { pain }\end{array}$ & \\
\hline Preop VAS back \& leg score & $6-10$ & "Moderate" & 7 & \\
\hline Postop VAS back pain score & 1 & 0 & 2 & \\
\hline Postop VAS leg pain score & 0 & 0 & 0 & \\
\hline Fluoroscopy time (mins) & 0.4 & 0.3 & 0.2 & 0.3 \\
\hline
\end{tabular}

ant = anterior; FU = follow-up.

In all 3 cases, given the otherwise extensive spondylotic changes throughout the lumbosacral spine and the fact that a dorsal approach with extensive facet resection would result in a potentially destabilizing decompression at L1-2 and would necessitate a multilevel fusion, a decision was made to undertake the surgery with direct access to the ventral elements via a minimally invasive lateral, retroperitoneal, direct transforaminal approach obviating the need for fusion.

\section{Postoperative Course}

All 3 patients reported significant improvement in their low-back pain and were fully ambulatory at discharge from the hospital in less than 24 hours after surgery. Postoperative follow-up ranged from 6 to 21 months. Assessment included monitoring of pain, numbness, and weakness and MRI studies. Numbness and dysesthetic pain was noted immediately postoperatively in all 3 patients as can be seen with the lateral transpsoas approach, and all patients had been counseled about this prior to surgery. The pain was well controlled with neuropathic medications such as gabapentin and pregabalin. This pain resolved after a few weeks, whereas the numbness, some of which had been present preoperatively, largely resolved by 6 months in cases 1 and 2 and by 2 months in case 3 . No new weakness was noted in any of the patients. In fact, the patient in case 2 noted improvement in his preoperative weakness, while the patient in case 3 had complete resolution of his left foot drop. Postoperative MRI obtained 1-6 months after surgery in all 3 patients showed no residual disc herniation with improved patency of the spinal canal and resolution of the stenosis (Fig. 4G-L). Further demographic, clinical, and outcomes parameters are listed in Table 1.

\section{Discussion}

Central and large paracentral disc herniations of the thoracolumbar and upper lumbar spine present a difficult management dilemma for the spine surgeon. The neural elements in this region are composed of the conus medul- laris and a large portion of the cauda equina. Thus, patients may present with a nebulous array of poorly localizing neurological symptoms that do not discretely correlate with distinct pathology in this anatomical region. Furthermore, the biomechanical considerations in this region are distinct from those in the lower lumbar spine. The facet joints are narrower and oriented in a more sagittal plane, and the region is juxtaposed between the more flexible lumbar and more rigid thoracic spine. These factors make the upper lumbar (and the thoracolumbar) spine more susceptible to instability from destabilizing procedures such as wide decompressive laminectomies as would be necessary to address central and large paracentral disc herniations in this region. Traditional posterior and posterolateral approaches to disc pathology in this region include dorsal laminectomy, ${ }^{5,12,14}$ laminectomy and transdural approach, ${ }^{4}$ and facetectomy and transforaminal lumbar interbody fusion. ${ }^{13,27}$ Minimally invasive transforaminal percutaneous endoscopic discectomies have been performed in this location in awake patients; ${ }^{1,25}$ however, the success rate for centrally located pathology was an abysmal $15 \%$ in one series. ${ }^{1}$ Anterior approaches to the upper lumbar spine for discectomy alone without corpectomy and fusion have not been described. Unsurprisingly, surgical treatment for lumbar disc herniations in this region using a myriad of approaches has yielded unsatisfactory outcomes as compared with those for lower lumbar disc herniations.,10,13,14,22,23 One could postulate that an ideal approach to central and large paracentral disc herniations in this region would allow for ventral decompression of the spinal canal without significant retraction of the thecal sac and would avoid a significant degree of bony facet resection while preserving the biomechanical integrity of the segment.

The retroperitoneal, transpsoas extreme lateral interbody fusion technique is a minimally invasive direct lateral approach designed to reach the ventral disc space for interbody fusion. ${ }^{18}$ However, the target region is typically the anterior third of the disc space in order to take advantage of the larger apophyseal surface with graft placement and to reduce subsidence rates. Targeting the anterior third 


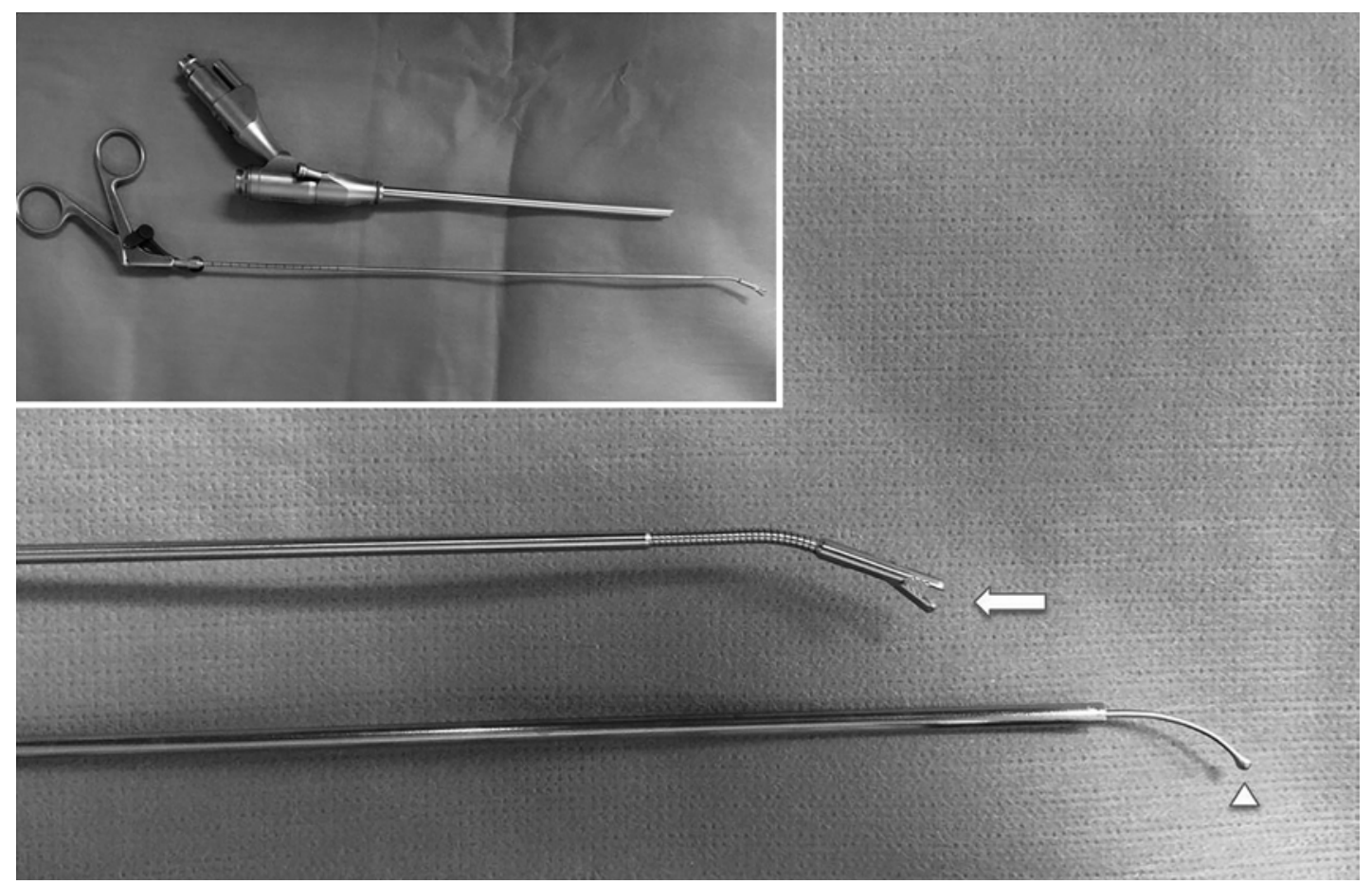

FIG. 5. Endoscopic flexible semi-bending grasper (arrow) and ball-tipped probe (arrowhead) used in microdissection. Grasper is shown alongside the endoscope (inset).

of the disc space also keeps the dissection away from critical neural elements such as the upper lumbar nerve roots and the lumbosacral plexus. While this approach has been modified for use in direct spinal canal decompression for burst fractures of the thoracolumbar spine and thoracic discectomies via a transthoracic approach, ${ }^{11,15}$ there are no reports of its use in paracentral and centrally placed upper lumbar discectomies without fusion. Moreover, transthoracic discectomy often requires partial vertebral body resection, which can necessitate a fusion. The challenge in utilizing this approach for central disc pathology in the upper lumbar spine is the need to work around the upper lumbar nerve roots and the psoas muscle.

Percutaneous endoscopic lumbar discectomy has recently evolved into a minimally invasive procedure performed in awake patients with lumbar disc pathology. ${ }^{26}$ The PELD approach is an oblique, posterior percutaneous approach that allows for direct endoscopic visualization of elements laterally within the neural foramen and less so of ventral central pathology. Thus, it is conducive to the removal of disc pathology situated paracentrally within the spinal canal or laterally within the neural foramen. However, because of a limited field of view through the endoscope and the oblique approach angle from a posterior approach, it may be less favorable for centrally situated disc pathology within the spinal canal. A recent report by Henmi et al. ${ }^{8}$ outlines the nuances of using this approach in a paracentrally situated L4-5 disc herniation. Given that it is a transforaminal approach, one would expect it to be better suited to the larger neural foramina of the upper lumbar spine. However, Ahn et al. ${ }^{1}$ describe a $57.6 \%$ success rate in lateral disc herniations at L1-2 and L2-3 and a $15 \%$ success rate for centrally located disc herniations for the same levels. ${ }^{1}$ Ruetten et al. describe an extreme lateral approach for transforaminal endoscopic discectomy, but it utilizes an oblique posterolateral approach in a prone patient, not a direct lateral, perpendicular, retroperitoneal transpsoas approach through the neural foramen. ${ }^{21}$ Disc herniations at L1-2 and L2-3 represented $1.7 \%$ and 5.2\%, respectively, of the total number of the 517 disc herniations treated. While they reported that $81 \%$ of their patients had no postoperative pain and that $5 \%$ showed no improvement, they did not specify the spinal levels at which the failures occurred. ${ }^{21}$

In this report, we describe an approach that is essentially a hybrid of the minimally invasive extreme or direct lateral, retroperitoneal transpsoas approach and the PELD to a central L1-2 disc herniation causing obliterative spinal stenosis. We have migrated the lateral retroperitoneal transpsoas approach posteriorly to the posterior third of the disc space at the neural foramen, and we have migrated the PELD approach anteriorly via a lateral transpsoas corridor to the neural foramen while also enlarging the neural foramen by foraminoplasty. This modified approach provides a direct lateral trajectory through the neural foramen, allowing for direct visualization of the ventral thecal sac and spinal canal without the need for retraction of the thecal sac or a destabilizing bony resection. Although the procedure was started with the endoscope, we enlarged the exposure with a minimally invasive retractor for better visualization with the operating microscope. Nonetheless, the long endoscopic semi-bendable graspers and flexible nerve hooks (Fig. 5) were necessary to safely perform the procedure given the long reach and increased working distance. The endoscope was also useful in examining the spinal canal in some patients after completing the discectomy. Intraoperative CT-guided navigation was used as an adjunct and 
was invaluable for precise localization within the exposure corridor and within the surgically enlarged neural foramen and the spinal canal. It was useful in further limiting the amount of bony resection and the dissection through the psoas muscle needed for access to the pathology of concern. Navigation was also used at the end of the case to confirm that the decompression had been carried clear across to the contralateral pedicle. We found that the use of CT-guided navigation greatly reduced the number of fluoroscopic localization images necessary to perform the case and thus decreased the radiation exposure of the surgeon and operating room staff. Nonetheless, in the absence of navigation, the procedure can be performed with fluoroscopic guidance. Given that the patient was under general anesthesia, electrophysiological monitoring (somatosensory evoked potentials, motor evoked potentials, electromyography) was performed throughout to ensure preserved integrity of the neural elements.

In our view, this approach confers several advantages over traditional open and minimally invasive posterior approaches: It provides a direct lateral perpendicular approach to the ventral thecal sac through a minimally invasive corridor, obviating the need for retraction of critical neural elements such as the conus and cauda equina. Additionally, access is gained without destabilizing bony resection, obviating the need for spinal fusion. The approach is feasible in the upper lumbar spine where the neural foramina are comparatively larger and the nerve to foramen ratio is favorable. To our knowledge, this approach has not been described in the literature for use in the upper lumbar spine. While further studies are necessary to determine whether the approach can be used at L2-3, we do not recommend its use for lower lumbar disc (L4-5) herniations as there is a higher risk of lumbosacral plexus injury given the smaller neural foraminal size and higher density of nerves.

A disadvantage of our approach, as compared with the PELD approach, is our use of general anesthesia given the lateral positioning of the patient and the retroperitoneal dissection. Awake patients undergoing PELD may provide better feedback on potential injury to upper lumbar nerve roots from retraction or manipulation. We also used a slightly larger $(3-4 \mathrm{~cm})$ incision for access than that used for PELD. A disadvantage of our procedure, compared with traditional transthoracic approaches, is that it may be less suited to calcified disc pathology given the small bony window and the increased oscillation of instruments used in dissection given the greater working distance $(140 \mathrm{~mm})$. Nonetheless, we did use the approach successfully in the patient in case 3 who had a partially calcified disc herniation. Our patients all experienced anterior thigh numbness and paresthesias following surgery as can be seen with the lateral transpsoas approach ${ }^{19,20,24}$ and probably from expansion of the tubular retractor; however, they resolved by $2-6$ months in all patients.

Finally, as described, our technique utilizes significant additional adjuvant resources-CT navigation, endoscopy, and electrophysiological monitoring-as compared with the traditional posterior discectomy with or without fusion. In the modern minimally invasive spine surgery operating room, it is not unusual to find specialized equipment for intraoperative image guidance and navigation, intraoperative CT scanning, and equipment for endoscopic spine surgery. Extreme lumbar interbody fusion procedures are routinely performed with electrophysiological monitoring, fluoroscopic guidance, and, at times, image guidance and navigation. Endoscopic surgery is performed with fluoroscopic guidance and an endoscope with a tower. While many of these resources may be readily available at a tertiary care academic center such as ours, they could be a significant resource burden on smaller hospitals. It is not our aim to suggest that intraoperative CT navigation, endoscopic spine techniques, and electrophysiological monitoring are necessary to perform routine lumbar disc surgery, but we have attempted to highlight how the combination of these technologies, when available, can enable minimally invasive spine surgeons with expertise in either endoscopic spine or minimally invasive extreme lateral surgery to approach large disc herniations in the upper lumbar spine differently.

\section{Conclusions}

In summary, our experience suggests the feasibility of a previously unreported approach to central and large paracentral disc herniations in the upper lumbar spine at the L1-2 level, utilizing a direct lateral approach through the neural foramen and with reasonably good outcomes. Further experience with a greater number of patients and at other upper lumbar spine levels will be necessary to define the safety and efficacy of this approach as a reliable alternative to other previously described approaches to similar pathology in this region.

\section{References}

1. Ahn Y, Lee SH, Lee JH, Kim JU, Liu WC: Transforaminal percutaneous endoscopic lumbar discectomy for upper lumbar disc herniation: clinical outcome, prognostic factors, and technical consideration. Acta Neurochir (Wien) 151:199206, 2009

2. Ahn Y, Lee SH, Park WM, Lee HY: Posterolateral percutaneous endoscopic lumbar foraminotomy for L5-S1 foraminal or lateral exit zone stenosis. Technical note. J Neurosurg 99 (3 Suppl):320-323, 2003

3. Albert TJ, Balderston RA, Heller JG, Herkowitz HN, Garfin SR, Tomany K, et al: Upper lumbar disc herniations. J Spinal Disord 6:351-359, 1993

4. Choi JW, Lee JK, Moon KS, Hur H, Kim YS, Kim SH: Transdural approach for calcified central disc herniations of the upper lumbar spine. Technical note. J Neurosurg Spine 7:370-374, 2007

5. Choudhury AR, Taylor JC, Worthington BS, Whitaker R: Lumbar radiculopathy contralateral to upper lumbar disc herniation: report of 3 cases. Br J Surg 65:842-844, 1978

6. Fontanesi G, Tartaglia I, Cavazzuti A, Giancecchi F: Prolapsed intervertebral disc at the upper lumbar level. Diagnostic difficulties. A report on 12 cases. Ital J Orthop Traumatol 13:501-507, 1987

7. Gutterman P, Shenkin HA: Syndromes associated with protrusion of upper lumbar intervertebral discs. Results of surgery. J Neurosurg 38:499-503, 1973

8. Henmi T, Terai T, Hibino N, Yoshioka S, Kondo K, Goda Y, et al: Percutaneous endoscopic lumbar discectomy utilizing ventral epiduroscopic observation technique and foraminoplasty for transligamentous extruded nucleus pulposus: technical note. J Neurosurg Spine 24:275-280, 2016 
9. Hsu K, Zucherman J, Shea W, Kaiser J, White A, Schofferman J, et al: High lumbar disc degeneration. Incidence and etiology. Spine (Phila Pa 1976) 15:679-682, 1990

10. Ido K, Shimizu K, Tada H, Matsuda Y, Shikata J, Nakamura $\mathrm{T}$ : Considerations for surgical treatment of patients with upper lumbar disc herniations. J Spinal Disord 11:75-79, 1998

11. Karikari IO, Nimjee SM, Hardin CA, Hughes BD, Hodges TR, Mehta AI, et al: Extreme lateral interbody fusion approach for isolated thoracic and thoracolumbar spine diseases: initial clinical experience and early outcomes. J Spinal Disord Tech 24:368-375, 2011

12. Kim DS, Lee JK, Jang JW, Ko BS, Lee JH, Kim SH: Clinical features and treatments of upper lumbar disc herniations. J Korean Neurosurg Soc 48:119-124, 2010

13. Kim JS, Lee SH, Moon KH, Lee HY: Surgical results of the oblique paraspinal approach in upper lumbar disc herniation and thoracolumbar junction. Neurosurgery 65:95-99, 2009

14. Lee DS, Park KS, Park MS: The comparative analysis of clinical characteristics and surgical results between the upper and lower lumbar disc herniations. J Korean Neurosurg Soc $\mathbf{5 4 : 3 7 9 - 3 8 3 , 2 0 1 3}$

15. Malham GM: Minimally invasive direct lateral corpectomy for the treatment of a thoracolumbar fracture. J Neurol Surg A Cent Eur Neurosurg 76:240-243, 2015

16. Moon KH, Lee SH, Kong BJ, Shin SW, Bhanot A, Kim DY, et al: An oblique paraspinal approach for intracanalicular disc herniations of the upper lumbar spine: technical case report. Neurosurgery 59 (4 Suppl 2):ONSE487-ONSE488, 2006

17. Oliveira L, Marchi L, Coutinho E, Pimenta L: A radiographic assessment of the ability of the extreme lateral interbody fusion procedure to indirectly decompress the neural elements. Spine (Phila Pa 1976) 35 (26 Suppl):S331-S337, 2010

18. Ozgur BM, Aryan HE, Pimenta L, Taylor WR: Extreme Lateral Interbody Fusion (XLIF): a novel surgical technique for anterior lumbar interbody fusion. Spine J 6:435-443, 2006

19. Pumberger M, Hughes AP, Huang RR, Sama AA, Cammisa FP, Girardi FP: Neurologic deficit following lateral lumbar interbody fusion. Eur Spine J 21:1192-1199, 2012

20. Rodgers WB, Gerber EJ, Patterson J: Intraoperative and early postoperative complications in extreme lateral interbody fusion: an analysis of 600 cases. Spine (Phila Pa 1976) 36:2632,2011

21. Ruetten S, Komp M, Godolias G: An extreme lateral access for the surgery of lumbar disc herniations inside the spinal canal using the full-endoscopic uniportal transforaminal approach-technique and prospective results of 463 patients. Spine (Phila Pa 1976) 30:2570-2578, 2005
22. Saberi H, Isfahani AV: Higher preoperative Oswestry Disability Index is associated with better surgical outcome in upper lumbar disc herniations. Eur Spine J 17:117-121, 2008

23. Sanderson SP, Houten J, Errico T, Forshaw D, Bauman J, Cooper PR: The unique characteristics of "upper" lumbar disc herniations. Neurosurgery 55:385-389, 2004

24. Sharma AK, Kepler CK, Girardi FP, Cammisa FP, Huang RC, Sama AA: Lateral lumbar interbody fusion: clinical and radiographic outcomes at 1 year: a preliminary report. J Spinal Disord Tech 24:242-250, 2011

25. Telfeian AE, Jasper GP, Oyelese AA, Gokaslan ZL: Technical considerations in transforaminal endoscopic spine surgery at the thoracolumbar junction: report of 3 cases. Neurosurg Focus 40(2):E9, 2016

26. Telfeian AE, Veeravagu A, Oyelese AA, Gokaslan ZL: A brief history of endoscopic spine surgery. Neurosurg Focus 40(2):E2, 2016

27. Wang J, Zhou Y, Zhang ZF, Li CQ, Zheng WJ, Huang B: Disc herniation in the thoracolumbar junction treated by minimally invasive transforaminal interbody fusion surgery. J Clin Neurosci 21:431-435, 2014

\section{Disclosures}

The authors report no conflict of interest concerning the materials or methods used in this study or the findings specified in this paper.

\section{Author Contributions}

Conception and design: Oyelese, Telfeian. Acquisition of data: Oyelese, Fridley, Choi, Telfeian. Analysis and interpretation of data: Oyelese, Choi. Drafting the article: Oyelese. Critically revising the article: Oyelese. Reviewed submitted version of manuscript: all authors. Approved the final version of the manuscript on behalf of all authors: Oyelese. Study supervision: Oyelese.

\section{Supplemental Information \\ Videos}

Video 1. https://vimeo.com/249528269.

\section{Correspondence}

Adetokunbo A. Oyelese: Brown University Alpert Medical School, Rhode Island Hospital, Providence, RI. aoyelese@ lifespan.org. 